\title{
Homogenization Theory and Digital Imaging: A Basis for Studying the Mechanics and Design Principles of Bone Tissue
}

\author{
S. J. Hollister ${ }^{1 *}$ and N. Kikuchi ${ }^{2}$ \\ ${ }^{1}$ Orthopaedic Research Laboratories, Section of Orthopaedic Surgery and \\ the Bioengineering Program, and ${ }^{2}$ Computational Mechanics Laboratory, \\ Department of Mechanical Engineering and Applied Mechanics, \\ University of Michigan, Ann Arbor, Michigan 48109
}

Received June 4, 1993/Accepted September 13, 1993

Bone tissue is a complex multilevel composite which has the ability to sense and respond to its mechanical environment. It is believed that bone cells called osteocytes within the bone matrix sense the mechanical environment and determine whether structural alterations are needed. At present it is not known, however, how loads are transferred from the whole bone level to cells. A computational procedure combining representative volume element (RVE) based homogenization theory with digital imaging is proposed to estimate strains at various levels of bone structure. Bone tissue structural organization and RVE based analysis are briefly reviewed. The digital image based computational procedure was applied to estimate strains in individual trabeculae (first-level microstructure). Homogenization analysis of an idealized model was used to estimate strains at one level of bone structure around osteocyte lacunae (second-level trabecular microstructure). The results showed that strain at one level of bone structure is amplified to a broad range at the next microstructural level. In one case, a zero-level tensile principal strain of $495 \mu \mathrm{E}$ engendered strains ranging between -1000 and $7000 \mu \mathrm{E}$ in individual trabeculae (first-level microstructure). Subsequently, a first-level tensile principal strain of $1325 \mu \mathrm{E}$ within an individual trabecula engendered strains ranging between 782 and $2530 \mu \mathrm{E}$ around osteocyte lacunae. Lacunar orientation was found to influence strains around osteocyte lacunae much more than lacunar ellipticity. In conclusion, the computational procedure combining homogenization theory with digital imaging can provide estimates of cell level strains within whole bones. Such results may be used to bridge experimental studies of bone adaptation at the whole bone and cell culture level. (c) 1994 John Wiley \& Sons, Inc.

Key words: homogenization theory - digital imaging • cortical bone $\bullet$ trabecular bone $\bullet$ osteocyte lacunae $\bullet$ bone mechanics

\section{INTRODUCTION}

Bone is a complex multiple tiered structure that has the ability to sense and adapt to its mechanical environment. Bone adaptation in response to mechanical stimuli plays a significant role in the outcome of many orthopaedic

\footnotetext{
* To whom all correspondence should be addressed.
}

and dental procedures, most notably artificial joint and tooth replacement. As early as 1867 Von Meyer (cited in ref. 47) suggested that trabecular bone was oriented along principal stress directions. Wolff ${ }^{55}$ expanded these observations suggesting that the internal structure of bone reflected its functional demands. Roux, in 1895 (cited in ref. 47), hypothesized that bone cells could sense and respond to mechanical stimuli. More recently, Brand ${ }^{6}$ hypothesized that bone adaptation was a feedback process in which bone cells monitor mechanical signals that have been filtered through the surrounding bone matrix. If the relevant mechanical signal is within an acceptable homeostatic range, the bone cells will not act on the signal. However, if the mechanical input signal is out of the homeostatic range, bone cells would alter the surrounding matrix filter such that the mechanical input signal was again brought within an acceptable range.

Testing hypotheses concerning bone adaptation has proved difficult because it is not known how mechanical loads are transferred from the whole bone level to bone cells. Experimental studies have therefore focused on the extremes of whole bone or isolated cell culture studies. At the whole bone level, researchers have measured strains on the outer surface of long bones under normal usage and after surgically induced overload. ${ }^{10,11,48}$ The results of these studies suggested that strains under normal usage ranged between 500 and 3200 microstrain $(\mu \mathrm{E})$. On the basis of these results, Frost ${ }^{19}$ hypothesized that strains less than $200 \mu \mathrm{E}$ would lead to bone resorption, strains between 200 and $2500 \mu \mathrm{E}$ would maintain bone, and strains greater than $2500 \mu \mathrm{E}$ would lead to bone apposition.

Whole bone studies provide insight into global responses to mechanical load but do not indicate levels of mechanical strain upon which cells sense and subsequently act. Studies stretching cultured bone cells and measuring their subsequent response have attempted to address the issue of how cells respond to direct mechanical strain. Harell et $\mathrm{al}^{23}$ applied $10,000 \mu \mathrm{E}$ to rat calvaria osteoblasts and found an increase in both prostaglandin $\left(\mathrm{PGE}_{2}\right)$ and cyclic adenosine monophosphate (cAMP) production. Yeh 
and $\operatorname{Rodan}^{56}$ applied between 50,000 and $100,000 \mu \mathrm{E}$ to rat osteoblasts by stretching the cells on a collagen sheet and also noted an increase in $\mathrm{PGE}_{2}$ production. Hasegawa et al. ${ }^{24}$ found an increase in cell proliferation when applying $20,000 \mu \mathrm{E}$ to mouse osteoblasts in culture. Murray and Rushton $^{42}$ noted a biphasic response when applying between 3000 and $28,000 \mu \mathrm{E}$ to mouse osteoblasts. Between 3000 and $7000 \mu \mathrm{E}$ no increase in $\mathrm{PGE}_{2}$ production or cell proliferation was noted. At $7000 \mu \mathrm{E}$ there was a jump in $\mathrm{PGE}_{2}$ production which leveled off until $28,000 \mu \mathrm{E}$ where another jump occurred. These and other cell culture studies have been recently reviewed by Burger and Veldhuijzen. $^{7}$

Cell culture studies have clearly demonstrated that bone cells can respond to direct mechanical stimulation. However, the strains applied in culture are much higher than those measured on whole bones. Strains of $20,000 \mu \mathrm{E}$ and higher, which have been applied in many cell culture studies, are high enough to fracture bone (ref. 19, p. 286). The strains applied in culture may be high enough to elicit a wound healing response from bone cells. It currently is not known, however, what strain levels bone cells see in vivo within the bone matrix. Strains on bone surfaces are measured at a scale much larger than and are far removed from bone cells surrounded by matrix. It is therefore difficult to extrapolate cell culture work to in vivo whole bone studies.

An intermediate step between cell culture and in vivo whole bone studies is the use of bone organ cultures. In a series of elegant experiments, El Haj et al. ${ }^{16}$ and Rawlinson et al. ${ }^{46}$ applied $5000 \mu \mathrm{E}$ of overall compressive strain to canine trabecular bone cores. El Haj et al. ${ }^{16}$ found an increase in serum $\mathrm{PGE}_{2}$ production $15 \mathrm{~min}$ after applying strain. Using immunolocalization techniques, Rawlinson et al. $^{46}$ found a breakdown product of prostacyclin $\mathrm{PGI}_{2}$ (6-keto- $\mathrm{PCF}_{1 \mathrm{a}}$ ) in osteocytes and both $\mathrm{PGI}_{2}$ and $\mathrm{PGE}$ in bone lining cells within trabecular bone cores subject to $5000 \mu \mathrm{E}$ overall strain. While these organ culture studies verified that cells within matrix produced PGE in response to strain as seen previously in cell culture, the cell level strains were not quantified.

The three experimental approaches for studying bone adaptation to mechanical stimuli (whole bone, organ culture, and cell culture) offer their own unique advantages and disadvantages. Whole bone studies offer the ability to study the global effects of bone cells on bone matrix but suffer from the disadvantage that the direct mechanical stimuli seen by cells cannot be quantified. Organ cultures offer the ability to directly load bone along with measuring cell response, but the mechanical stimuli seen by an individual cell again has not been quantified. Also, the modification of bone matrix by bone cells cannot be reproduced in culture. Cell culture studies offer the ability to directly control the mechanical stimuli applied to cells along with measuring cell response but suffer from the lack of normal bone matrix surrounding bone cells. Furthermore, it is not known how mechanical strains applied to bone cells in vitro compare to strains which bone cells experience in vivo under normal loading.

To better understand bone adaptation to mechanical stimuli, it is necessary to link whole bone, organ culture, and cell culture experiments. Knowing strains on bone cells within bone matrix would allow this linkage. For example, if it were known that bone cells subject to a given amount of strain in vivo caused a certain change in bone matrix, this same amount of strain could be applied to both organ and cell cultures to study the cellular biochemical response. Measuring cell level strains within bone matrix has proved impossible to date since strains can only be measured on the surface of structures using strain gauges or optical techniques. The natural structures surrounding the cell would have to be destroyed to measure the cell strain. Perhaps the most feasible approach for estimating cell level strains is computational modeling of the multiple level bone structures. In this article, we present one multilevel composite modeling approach to estimate cell strains for whole bones. First, bone tissue structure and composite material theories used to model this structure are reviewed. Second, the homogenization approach we currently use along with estimates of cell lacunae level strains is presented.

\section{BACKGROUND}

\section{Bone Tissue Structural Organization}

Bone tissue is classified into two broad types based on its macroscopic porosity. Cortical bone, also called compact bone, contains about $10 \%$ pore volume and accounts for $80 \%$ of all skeletal mass. ${ }^{35}$ Cortical bone forms the outer shell of all long bones. Trabecular bone, also called cancellous bone, is much more porous than cortical bone. Trabecular bone porosity is about $75 \%$ on average but may range between $50 \%$ and $90 \%{ }^{35}$ Trabecular bone fills the end of long bone and is contained within cortical bone. Each type of bone tissue contains at least three definable levels of microstructure.

The first microstructural level of cortical bone contains tubular structures called osteons or haversian systems sitting within a matrix of interstitial lamellae (Fig. 1). A blood vessel runs through the center of the osteon. Osteon diameters are typically between 200 and $300 \mu \mathrm{m}$ with the interior haversian canal diameter ranging from 50 to $90 \mu \mathrm{m}^{41}$ The inner blood vessel diameter is typically $15 \mu \mathrm{m} .{ }^{41}$ The second microstructural level (Fig. 2) contains ellipsoidal shaped voids called lacunae typically $10 \mu \mathrm{m}$ in radius which house bone cells called osteocytes. The lacunae are within $150 \mu \mathrm{m}$ radius ${ }^{41}$ of the inner blood vessel because of nutrition diffusion requirements. The osteonal wall consists of concentric sheets called lamellae which are between 7 and $10 \mu \mathrm{m}$ thick. Osteons are bonded to surrounding interstitial bone by cement lines which are about 1 to $50 \mu \mathrm{m}$ thick. ${ }^{51}$ The third microstructural layer consists of 


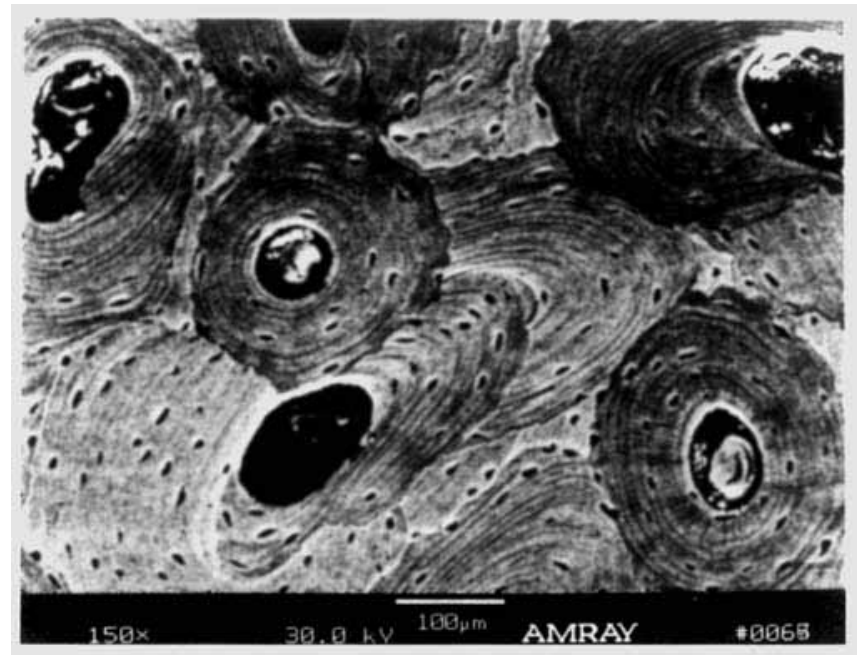

Figure 1. Backscattered scanning electron micrograph of first-level cortical bone microstructure. This first-level microstructure consists of osteons (irregular shaped ovals of differing darkness) and interstitial bone (bone between the osteons). The dark circles of about $100 \mu \mathrm{m}$ in diameter are the haversian canals containing blood vessels. (Photograph courtesy of Karl Jepsen.)

the collagen fibers bonded to plate-like hydroxyapatite mineral crystals ${ }^{53}$ within a single lamella. The orientation of the collagen-mineral composite within and between successive lamellae is currently the focus of much debate. 1,2,22,40,53 Some authors believe that collagen fibers have a predominant orientation which gradually changes between lamellae. Marotti and Muglia, ${ }^{40}$ however, suggested that lamellae consisted of either loosely or densely packed collagen fibers with no preferred orientation. It should be noted that all dimensions reported here are for human bone and that dimensions of osteons, lamellae, and lacunae may differ for bone from other species.

The first microstructural level of trabecular bone consists of a network of plate- and rod-like structures called

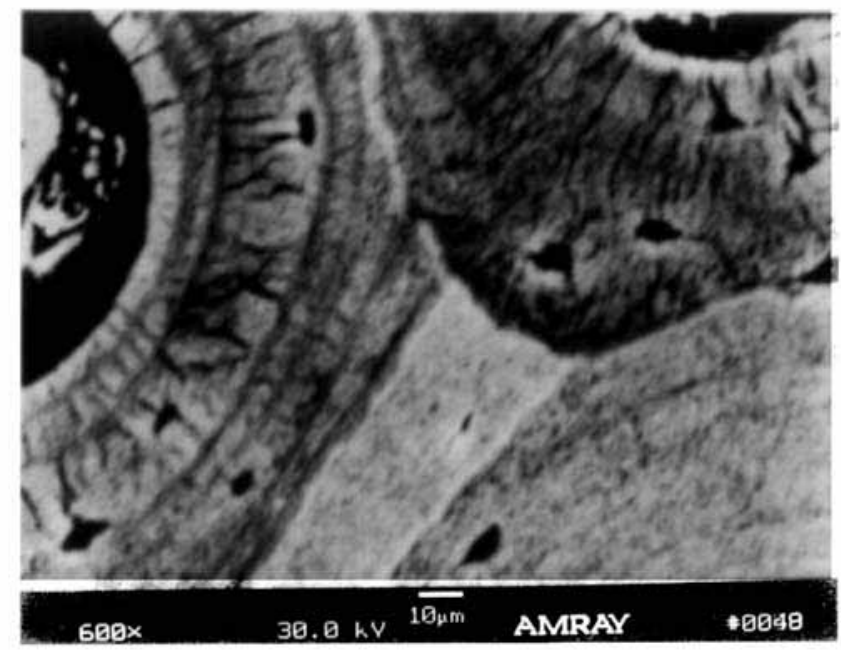

Figure 2. Backscattered scanning electron micrograph of second-level cortical bone microstructure. The smaller ellipsoidal shapes about $10 \mu \mathrm{m}$ long are lacunae. White lines separate osteons. Small dark striations are canaliculi. (Photograph courtesy of Karl Jepsen.) trabeculae (Fig. 3). Unlike osteons, trabeculae rarely contain blood vessels and are thereby generally less than $200 \mu \mathrm{m}$ thick because of diffusion requirements. ${ }^{35}$ At the second level of microstructure trabeculae contain lamellae which are not concentric-like osteonal lamellae but rather are aligned the length of the trabeculae. These lamellae are grouped together in trabeculae packets. ${ }^{35}$ Lacunae in trabeculae are slightly larger and more densely packed than in osteons. ${ }^{8}$ As with cortical bone, the third level of microstructure in trabecular bone consists of collagen fibers bonded to mineral crystals. A summary of structural organizations for both cortical and trabecular bone is shown in Table I.

A nonstructural but critical component of bone tissue is the bone cells themselves. There are three basic types of bone cells called osteoblasts, osteocytes, and osteoclasts. Of these osteoblasts and osteoclasts reside on bone surfaces while osteocytes reside internally within the bone matrix. Osteoblasts deposit both collagen and mineral to form bone structure. During bone formation osteoblasts are plump cuboidal cells. Following bone formation, osteoblasts become flat and elongated, covering the bone surfaces. Osteocytes evolve from osteoblasts which are entrapped in bone matrix during the process of bone deposition. These cells are believed by many to sense the mechanical environment within the bone tissue. Osteoclasts resorb bone mineral and collagen. Osteoclasts and osteoblasts act together to maintain bone structure through a process called remodeling. ${ }^{19}$ Osteoclasts first resorb bone from the bone surface, leaving a resorption pit. This pit is then filled by bone deposited by osteoblasts. In special cases, such as growth or repair of damaged tissue, osteoblasts may deposit bone and osteoclasts may resorb bone on separate surfaces. This process is called modeling. ${ }^{19}$

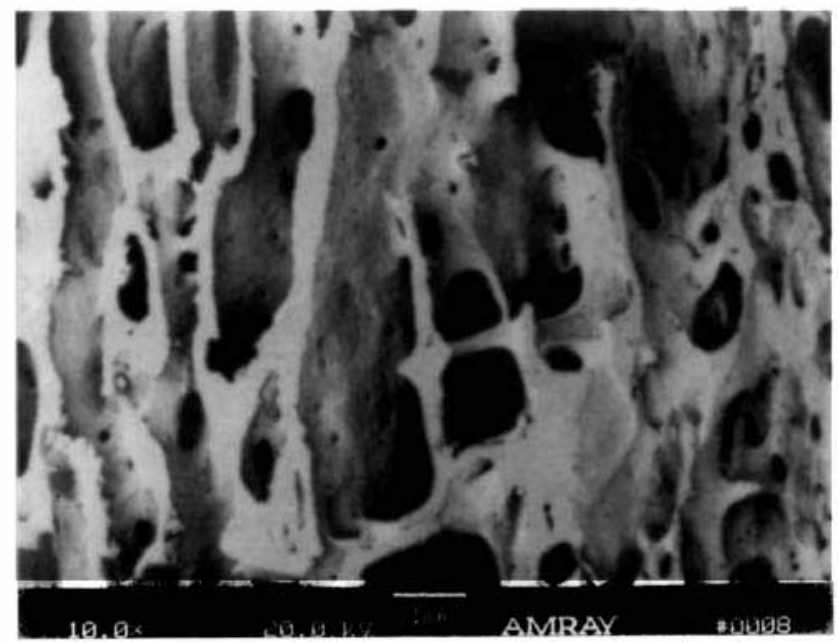

Figure 3. Backscattered scanning electron micrograph of first-level trabecular bone microstructure. At this level trabecular bone consists of a network of rods and plates, approximately 100 to $200 \mu \mathrm{m}$ thick and over $1000 \mu \mathrm{m}$ long. (Photograph courtesy of Karl Jepsen.) 
Table I. Trabecular and cortical bone structural organization along with approximate physical scales.

\begin{tabular}{|c|c|c|c|c|}
\hline Level & Cortical structure & Trabecular structure & Size range $(\mu \mathrm{m})$ & $\eta$ \\
\hline 0 & solid material & solid material & $>3000$ & - \\
\hline 1 & osteons, interstitial bone & individual trabeculae & $100-300$ & $\leq 0.1$ \\
\hline 2 & lamallae, lacunae, cement lines & lamellae, lacunae, cement lines & $3-20$ & $\leq 0.1$ \\
\hline 3 & collagen-mineral composite & collagen-mineral composite & $0.06-0.4$ & $\leq 0.1$ \\
\hline
\end{tabular}

The parameter $\eta$ is a ratio between the level $i$ and the next most macroscopic level $i-1$. This parameter is found implicitly in homogenization RVE analysis. Decreasing $\eta$ will produce decreasing error.

\section{Representative Volume Element Analysis for Composite Materials}

Due to its complex geometry, bone tissue is generally analyzed using numerical techniques, of which the most widely used is the finite element method (FEM). However, standard finite element analysis codes running even on modern large capacity supercomputers cannot analyze each structural entity from the whole bone to the bone cell level. Therefore, each structural level must be analyzed separately. This approach is known in the engineering composite material literature as representative volume element (RVE) analysis. ${ }^{25,27}$ In RVE analysis, representative sections of a structural level are analyzed under assumed boundary conditions to calculate the average or effective properties of that section. This is known as the local analysis. The local analysis provides a matrix relating local strains $\left\{\boldsymbol{\varepsilon}_{i}\right\}$ to global strains $\left\{\varepsilon_{i-1}\right\}$ :

$$
\left\{\varepsilon_{i}\right\}=\left[M_{i}\right]\left\{\varepsilon_{i-1}\right\}
$$

where the subscript $i$ denotes the current structural level being analyzed, $i-1$ denotes the next most macroscopic level, and $\left[M_{i}\right]$ is denoted as the local structure matrix. ${ }^{30}$ The average or effective stiffness may then be calculated from the local structure matrix:

$$
\left[C_{i-1}\right]=\frac{1}{V_{\mathrm{RVE}}} \int_{V_{\mathrm{RVE}}}\left[C_{i}\right]\left[M_{i}\right] d V_{\mathrm{RVE}}
$$

where $\left[C_{i-1}\right]$ is the stiffness of the $i-1$ structural level, $\left[C_{i}\right]$ is the stiffness of the $i$ th structural level, $V_{\mathrm{RVE}}$ is the RVE volume, and $\left[M_{i}\right]$ is the $i$ th level local structure matrix. The next most macroscopic level can then be analyzed using the previously calculated effective properties. This is known as the global analysis and provides the global strains to calculate the local strains using Eq. (2). The entire procedure is illustrated in Figure 4.

The RVE concept may be applied to estimate strains on multiple structural levels. However, since the in situ RVE boundary conditions are not known, the assumed boundary conditions can only provide an estimate of both effective properties and local strains. The accuracy of these estimates depends significantly on the assumed boundary conditions. The most widely used RVE approach is to apply traction or displacement boundary conditions to the RVE. This approach, denoted here as the standard mechanics approach, has been used frequently for analyzing bone tissue micromechanics. ${ }^{5,15,21,28,45,54}$ It can be shown using the minimum potential energy and minimum complementary energy principles of mechanics that displacement boundary conditions will provide an upper bound on effective stiffness while traction boundary conditions will provide a lower bound. ${ }^{27}$ Bounds on local strain errors have not been derived. A modification of the standard mechanics approach using assumed stress of strain distributions based on variational methods ${ }^{26}$ have also been used to estimate cortical bone stiffness based on a fiber matrix idealization. ${ }^{36}$

\section{Homogenization Theory}

Homogenization theory is another RVE approach developed by applied mathematicians both in the West ${ }^{3,37,39,49,50}$ and in the former Soviet Union (see ref. 4 for a review). There are three basic assumptions in homogenization theory. First, it is assumed that the total displacement of a microstructured material may be represented by an asymptotic expansion:

$$
\left\{u_{\eta}\right\}=\left\{u_{0}\right\}+\eta\left\{u_{1}\right\}+\eta^{2}\left\{u_{2}\right\}+\cdots
$$

where $\left\{u_{\eta}\right\}$ is the total displacement, $\left\{u_{0}\right\}$ is the most macroscopic level displacement, $\left\{u_{i}\right\}$ represents displacements for increasingly more microscopic levels, and $\eta$ is the relative scale size between two scalewise adjacent structural levels. It can clearly be seen that the multiple structural levels of bone can be fit into the asymptotic expansion framework. The second assumption is that the coordinates at each level are related through the parameter $\eta$, as illustrated in Figure 5. The coordinates in $x_{i}$ on the microscopic level are much finer than the coordinates on

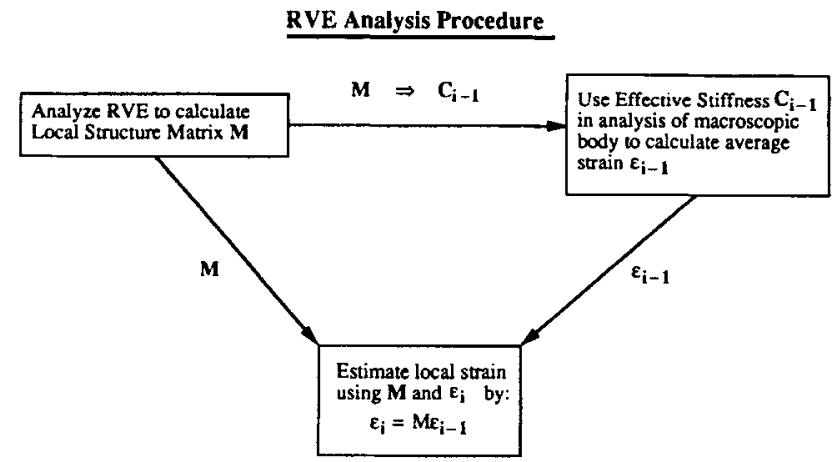

Figure 4. Illustration of the RVE analysis procedure. Local analysis gives the local structure matrix $M$ and the effective stiffness $C_{i-1}$. Global analysis yields the strains $\varepsilon_{i-1}$. Postprocessing of $M$ with $\varepsilon_{i-1}$ yields the strains $e_{i}$ at the current microstructure level. 
$x_{i-1}$, the more macroscopic level. The coordinates $x_{i}$ are thus related to the $x_{i-1}$ coordinates by

$$
x_{i}=\frac{x_{i-1}}{\eta}
$$

Values of $\eta$ for different levels of bone tissue are suggested in Table I. Although $\eta$ does not appear explicitly in the final formulation, smaller values of $\eta$ give a better approximation for the RVE analysis. The total gradient of a function with respect to all structural levels may then be written as the sum of gradients at each separate level modified by the scale factor $\eta$ :

$$
\frac{\partial}{\partial\{x\}}=\frac{\partial}{\partial\left\{x_{0}\right\}}+\frac{1}{\eta} \frac{\partial}{\partial\left\{x_{1}\right\}}+\frac{1}{\eta^{2}} \frac{\partial}{\partial\left\{x_{2}\right\}}+\cdots
$$

The third assumption made in homogenization theory is that the RVE boundary displacements are periodic, that is, the displacements on one RVE side are equal to those on the opposite side. Hollister and Kikuchi ${ }^{31}$ have shown that homogenization theory gives more accurate estimates of both effective stiffness and local strain than standard mechanics approaches for composites with periodic microstructure.

Recent analysis of bone mechanics using homogenization theory has been done by two groups. Hollister and co-workers ${ }^{29,30}$ analyzed trabecular bone using both idealized models and models constructed directly from threedimensional (3-D) digitized images of trabecular bone. They found that idealized models showed trends of how structural characteristics affected mechanical properties but that digital image based models produced much more accurate effective stiffness estimates. Crolet et al. ${ }^{14}$ modeled cortical bone at first two microstructural levels, including osteons and lamellae. They predicted macroscopic effective stiffness values which were similar to experimental values.

\section{Generating Computer Models of Bone Tissue Structure}

Computational analysis of bone tissue microstructure using any RVE approach requires construction of a finite element method (FEM). Most models of bone tissue structure have been based on idealizations. For example, the first-level microstructure of trabecular bone is often idealized as a porous cellular material. ${ }^{5,21,30,45}$ The first-level osteonal microstructure of cortical bone has been idealized as a matrix containing an osteonal cylinder with

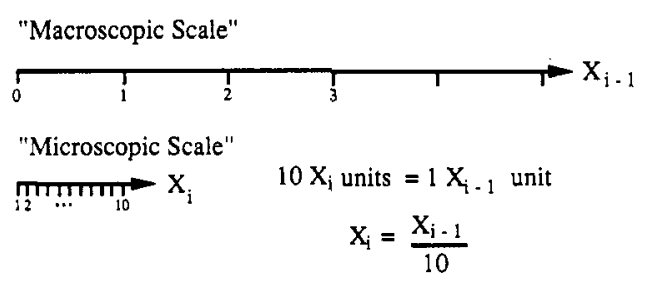

Figure 5. Illustration of the relationship between different coordinate levels and definition of the parameter $\eta$. Ten units on the microscopic scale $X_{i}$ are equal to 1 unit on the macroscopic scale $X_{i-1}$. Thus, $X_{i}=X_{i-1} / 10$, which means that $\eta=1 / 10$, or 0.1 an interior cylindrical hole. ${ }^{28}$ Idealized models are relatively straightforward to construct using modern solid modeling programs. These models can be used to parametrically test how different structural features may influence the mechanical behavior of bone tissue, thereby giving insight into how cells may organize structural components to best carry load. However, since bone tissue structure is very irregular and variable, idealized models do not give very accurate estimates for specific bone tissue structures. The use of idealized models for analyzing and interpreting experimental data on bone adaptation is limited.

Recent developments of 3-D imaging techniques for trabecular bone microstructure either based on nondestructive computed tomography ${ }^{17}$ or by sequential sectioning and two-dimensional (2-D) imaging ${ }^{43}$ have given rise to digital image based finite element analysis of bone microstructure..$^{20,29,32,52}$ Digital image based finite element analysis constructs 3-D finite element models by directly converting image voxels into 3-D brick finite elements. Mesh generation of complex microstructures can thus be done in minutes or hours instead of days or months. This is especially important when hundreds of finite element RVE models must be analyzed to interpret experimental data. The repeated regular geometry of elements within a digital image based mesh are well suited for element-by-element preconditioned conjugate gradient (EBE-PCG ${ }^{9,18}$ ) iterative solution techniques. These techniques solve finite element equations locally on the element level without need for assembly of a global stiffness matrix, which means that meshes with hundreds of thousands of elements may be solved on engineering workstations.

There are two major drawbacks to digital image based finite element analysis. First, the jagged edges at the boundary between dissimilar materials introduce artificial stress concentrations. These stress concentrations cause numerical oscillations which are not present in smooth finite element meshes. Hollister and Riemer, ${ }^{32}$ however, found that these numerical oscillations were present only in localized regions near material boundaries and that digitized finite element meshes produced solutions very similar to smooth finite element analysis. Digital image finite element meshes produce reliable solutions over most of the mesh except at material boundaries. In these regions, techniques for smoothing the solutions are needed.

The second difficulty in digital image based finite element analysis is relating the voxel density from the digitized image to a material stiffness for the finite element analysis. Analysis of the first-level trabecular bone microstructure requires thresholding techniques which only need delineate between bone tissue and marrow. Each trabecula is treated as a homogeneous isotropic material which is assigned experimentally measured properties. Using this approach, Hollister et al. ${ }^{29}$ found good agreement between predicted and experimental effective stiffness values using individual trabecular stiffness results reported by Choi et al. ${ }^{12}$ When more than two material phases are present, more sophisticated image thresholding techniques are needed to delineate 
between the phases. Furthermore, experimental data must be generated such that material stiffness values may be assigned to each threshold voxel density. The general procedure of digital image based homogenization analysis is as follows:

1. Threshold digital image voxels into separate materials.

2. Convert 3-D image voxels to 3-D solid finite elements.

3. Select element material identification based on thresholded voxel density.

4. Set element material property based on experimental measures relating stiffness to voxel density.

5. Perform homogenization analysis using EBE-PCG techniques.

\section{METHODS}

\section{Homogenization Analysis}

Using Eqs. (3) and (5) allows the total strain at the smallest microstructural level to be written as

$$
\{\varepsilon\}=\left\{\varepsilon_{0}\right\}+\left\{\varepsilon_{1}\right\}+\left\{\varepsilon_{2}\right\}+\cdots
$$

where $\{\varepsilon\}$ is the total strain at the smallest microstructural scale, $\left\{\varepsilon_{0}\right\}$ is the most global or average strain, $\left\{\varepsilon_{1}\right\}$ is a perturbation in strain due to microstructure at the first level, $\left\{\varepsilon_{2}\right\}$ is a perturbation in strain due to microstructure at second level, and so on. Note that the total strain at any level results from the sum of the strain perturbation at that level and those above that level. For example, the total strain at the first level, individual trabeculae, is the sum of the solid material zero-level strain $\left\{\varepsilon_{0}\right\}$ and the firstlevel strain perturbation $\left\{\varepsilon_{1}\right\}$. Likewise, strain at the lacunar level is the sum of the solid material zero-level strain $\left\{\varepsilon_{0}\right\}$, the individual trabeculae first-level strain perturbation $\left\{\boldsymbol{\varepsilon}_{1}\right\}$, and the lacunar second-level perturbation strain $\left\{\boldsymbol{\varepsilon}_{2}\right\}$. Equation (6) is substituted into the standard weak form of the elasticity equations. This generates two equilibrium equations, one for the more microscopic level and one for the more macroscopic level (for details, see ref. 29 or 30). For instance, when considering trabeculae to be homogeneous material, the trabecular architecture would be the more microscopic level and the representation of trabecular bone as a solid would be the more macroscopic level. The equilibrium equation on the more microscopic level is

$$
\int_{V_{\mathrm{RVE}}}\{\hat{\varepsilon}\}^{T}\left[C_{i}\right]\left\{\varepsilon^{* k}\right\} d V_{\mathrm{RVE}}=\int_{\mathrm{V}_{\mathrm{RVE}}}\{\hat{\varepsilon}\}^{T}\left\{C_{i}^{k}\right\} d V_{\mathrm{RVE}}
$$

where the superscript $T$ denotes the transpose of the vector $\{\hat{\boldsymbol{\varepsilon}}\},\left[C_{i}\right]$ is the $i$ th microscopic level material stiffness matrix, $\{\hat{\boldsymbol{\varepsilon}}\}$ is the microscopic virtual strain vector, $\left\{\varepsilon^{* k}\right\}$ is the microscopic characteristic strain vector, and $\left\{C_{i}^{k}\right\}$ is the $k$ th column of the $i$ th microscopic material stiffness matrix. Equation (6) is solved six times, once for each column of
$\left[C_{i}\right]$. The right-hand side of Eq. (7) is a volume distributed stress over the RVE. In addition, opposite sides of the RVE are assumed to displace equally under the volume distributed stress in Eq. (7). The corner nodes of the RVE model are fixed to prevent rigid body motion. Note that Eq. (7) is solved for every structural level except the zeroor solid material level. The local structure matrix can then be calculated from six characteristic strain vectors as

$$
[M]=[I]-\left[\left\{\varepsilon^{* 1}\right\}\left\{\varepsilon^{* 2}\right\}\left\{\varepsilon^{* 3}\right\}\left\{\varepsilon^{* 4}\right\}\left\{\varepsilon^{* 5}\right\}\left\{\varepsilon^{* 6}\right\}\right]
$$

where $[M]$ is the local structure matrix, $[I]$ is the identity matrix, and the $\left\{\varepsilon^{* k}\right\}$ are the six $6 \times 1$ microscopic characteristic strain vectors forming a $6 \times 6$ matrix. Local structure matrices are calculated at every structural level except the zero level. The effective stiffness is calculated using Eq. (2). At the most macroscopic level, the governing equilibrium equation under applied boundary conditions is

$$
\begin{aligned}
& \int_{\Omega}\{\hat{\varepsilon}\}^{T}\left[C_{0}\right]\left\{\varepsilon_{0}\right\} d \Omega= \\
& \quad \int_{\Gamma_{t}}\{v\}^{T}\{t\} d \Gamma t+\int_{\Gamma_{d}}\{v\}^{T} \lambda(\{u\}-\{g\}) d \Gamma_{d}
\end{aligned}
$$

where $T$ denotes the transpose of a given vector, $\{\hat{\varepsilon}\}$ is again a virtual strain vector, $\left[C_{0}\right]$ is the most macroscopic effective stiffness, $\left\{\varepsilon_{0}\right\}$ is the most macroscopic strain vector, $\{v\}$ is the virtual displacement vector, $\lambda$ is a penalty parameter (see ref. 38), $\{u\}$ is the displacement vector, $\{g\}$ is a prescribed displacement, $\{t\}$ is an applied traction, $\Omega$ is the global domain, $\Gamma_{t}$ is the boundary along which tractions are prescribed, and $\Gamma_{d}$ is the boundary along which displacements are prescribed. Solving Eq. (9) gives the solid material or zero-level strain $\left\{\varepsilon_{0}\right\}$. Both Eqs. (7) and (9) are solved using the FEM. Strains are calculated at the first level or trabecular level by transformation of $\left\{\varepsilon_{0}\right\}$ with the first-level local structure matrix:

$$
\left\{\varepsilon_{\text {trab }}\right\}=\left[M_{1}\right]\left\{\varepsilon_{0}\right\}
$$

Strains are calculated at the second level or lacunar level by transformation of the average trabecular strain in the lacunar region with the second-level structure matrix:

$$
\left\{\varepsilon_{\text {lacunar }}\right\}=\left[M_{2}\right]\left\{\varepsilon_{\text {trab }}\right\}
$$

Note that Eqs. (10) and (11) can be combined to directly calculate the second- or lacunar level strain from the zerolevel strain by

$$
\left\{\varepsilon_{\text {lacunar }}\right\}=\left[M_{2}\right]\left[M_{1}\right]\left\{\varepsilon_{0}\right\}
$$

\section{Computational Models for First- and Second-Level Trabecular Microstructure}

This study utilized both digital image based models and idealized models to analyze how load is transferred in trabecular bone from the whole bone level to the osteocyte lacunae. Trabecular bone subject to controlled implant loads from the study by Hollister and Goldstein ${ }^{33}$ was considered since the global boundary conditions were known. 
First-level trabecular bone microstructure in this case was analyzed using digital image based homogenization finite element analysis. The models were constructed by converting digital voxels from 3-D micro-computerized tomography scans directly into linear brick finite elements. A total of 10 regions having a volume of $4 \times 4 \times 4 \mathrm{~mm}$ $\left(64 \mathrm{~mm}^{3}\right)$ were scanned within the femur. The RVE size was chosen to be $1 \times 1 \times 1 \mathrm{~mm}$, giving a total of 640 analyses involving 8000 solid elements per mesh. A typical digitized finite element mesh for the homogenization analysis is shown in Figure 6.

Idealized 3-D models of osteocyte lacunae constituting second-level trabecular microstructure were created to estimate strains surrounding osteocytes within trabeculae tissue. The osteocyte lacunae were modeled as ellipsoidal voids within an isotropic bone matrix using the solid modeling program PATRAN (PDA Engineering, Costa Mesa, CA). Although microstructure at this level also contains canaliculi, our interest was to estimate how lacunae could affect strain distributions within the bone matrix. Therefore, canaliculi were not included in the idealized model. The total RVE volume was $50 \times 50 \times 50 \mu \mathrm{m}$ or $.000125 \mathrm{~mm}^{3}$. Two ellipsoidal voids having major to minor radius ratios of 1.5:1 (Fig. 7) and 2:1 were analyzed. The voids occupied approximately $5 \%$ (1.5:1 ratio) and $7 \%$ (2:1 ratio) of the total RVE volume, respectively. These lacunae volumes were chosen to approximately match results by Cané et al. ${ }^{8}$ who found that osteocyte lacunae occupied about $4 \%$ of the bone matrix volume in trabecular bone of canine proximal femurs. The bone matrix surrounding the lacunae were assumed to be isotropic with Young's modulus $E=3.9$ gigapascals $(\mathrm{GPa})$ and Poisson's ratio $v=0.3$. Although bone matrix is most likely anisotropic

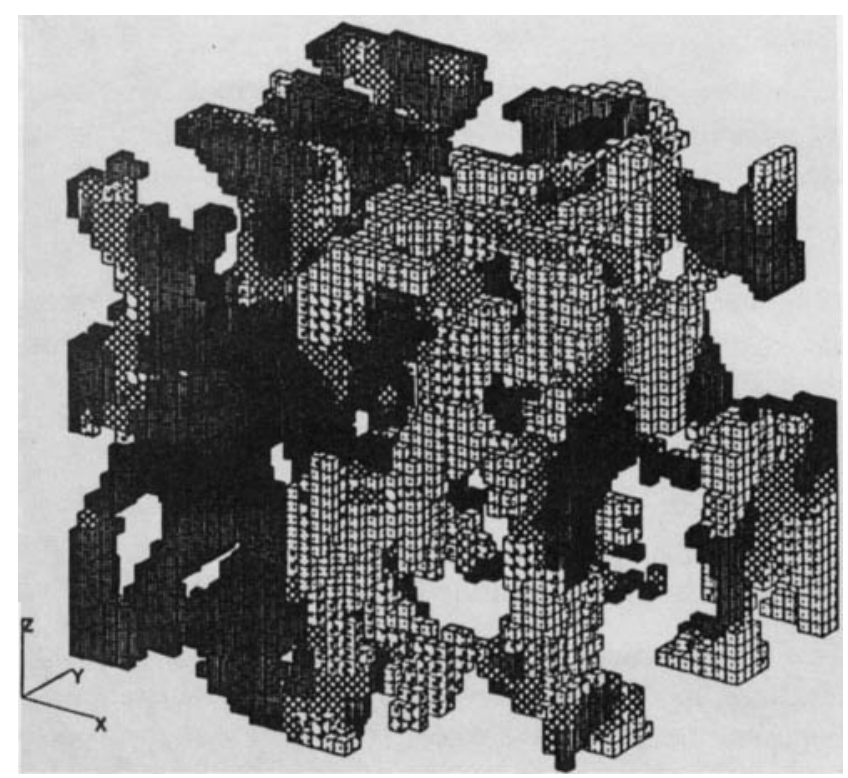

Figure 6. A representative digital image based finite element mesh of first-level trabecular bone microstructure. The region is $2 \mathrm{~mm}$ on a side with voxel/element lengths of $50 \mu \mathrm{m}$.

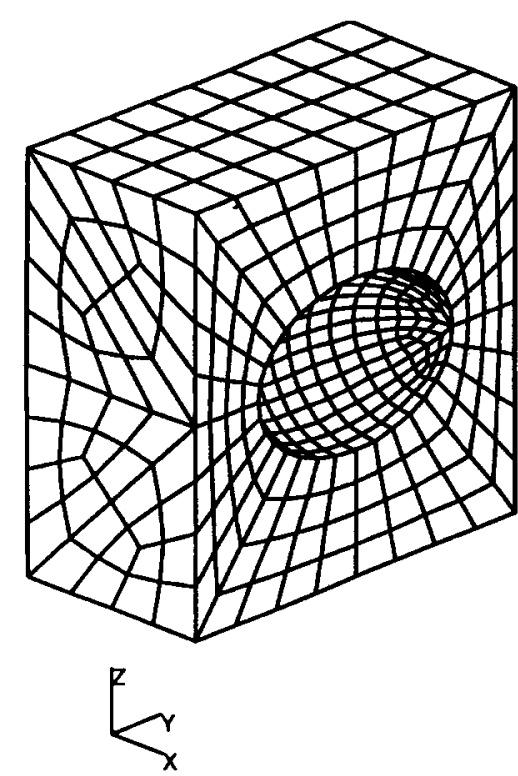

Figure 7. Finite element mesh of an idealized osteocyte lacunae sitting within an isotropic bone matrix. Only half of the total RVE mesh is shown to illustrate the ellipsoidal geometry of the lacunae.

due to its microstructure, nothing is known about the bone lamellar stiffness for trabecular bone. Therefore, the simplest isotropic model was used as an approximation for this preliminary study. The canine bone lamellar tissue stiffness magnitude was assumed to be similar to the canine tissue stiffness magnitude measured by Choi and Goldstein. ${ }^{13}$ Homogenization analysis of the lacunar models yielded both the effective stiffness matrix and local structure matrix. The lacunar level strains were calculated by postprocessing the first-level microstructure strains with the local structure matrix from the lacunar model. Strains within one $50 \times 50 \times$ $50-\mu \mathrm{m}$ region from a trabecula were chosen to calculate the lacunar level strains. Lacunar orientations aligned with the first-level microstructure principal strain directions and at an arbitrary angle were analyzed.

\section{RESULTS}

\section{First-Level Trabecular Microstructure}

The first-level trabecular microstructure leads to a completely anisotropic zero-effective stiffness for the region analyzed:

$\left[C_{0}\right]=$

$$
\left[\begin{array}{rrrrrr}
397.0 & 62.2 & 101.2 & -2.6 & 2.4 & -11.4 \\
& 246.1 & 67.2 & -1.5 & -0.4 & -7.8 \\
& & 478.8 & -5.9 & 2.9 & -3.5 \\
& & & 78.6 & -7.1 & 0.9 \\
& \text { SYM } & & & 141.8 & -3.8 \\
& & & & & 70.4
\end{array}\right]
$$

where all stiffness values are given in megapascals (MPa). Under the uniaxial implant loads on the canine distal femur 
as reported by Hollister and Goldstein, ${ }^{33}$ the zero-level (whole bone level) principal strains for the region analyzed were

$$
\left\{\varepsilon_{0}\right\}=\left\{\begin{array}{r}
-765 \mu E \\
-192 \mu E \\
495 \mu E
\end{array}\right\}
$$

where $\mu \mathrm{E}$ denotes microstrain. These zero-level strains engendered first-level maximum principal strains ranging from $-1000 \mu \mathrm{E}$ to over $7000 \mu \mathrm{E}$. Although maximum principal tissue level strains most frequently centered around the zero-level strain of $495 \mu \mathrm{E}$, a significant portion were over $1000 \mu \mathrm{E}$ (Fig. 8). First-level minimum principal strains ranged between $-7500 \mu \mathrm{E}$ in compression to $2500 \mu \mathrm{E}$ in tension.

\section{Second-Level Trabecular Microstructure}

The architecture of the second-level microstructure had a very small influence on the first-level effective stiffness. Due to the ellipsoidal void, the effective stiffness was orthotropic. However, the effective stiffness constants for the bone matrix containing the lacunae were only $5 \%$ less than the assumed stiffness of the bone matrix itself.

The first-level principal strains from the region used to calculate the lacunar level strains were

$$
\left\{\varepsilon_{\text {trab }}\right\}=\left\{\begin{array}{r}
1325 \mu E \\
287 \mu E \\
80 \mu E
\end{array}\right\}
$$

The first-level principal strains in this case were all tensile. The strains engendered from the first level microstructure depended significantly on the lacunar orientation relative to first-level principal strain directions. Lacunae at an arbitrary orientation were subject to a nonuniform strain distribution while lacunae oriented along the principal strain directions

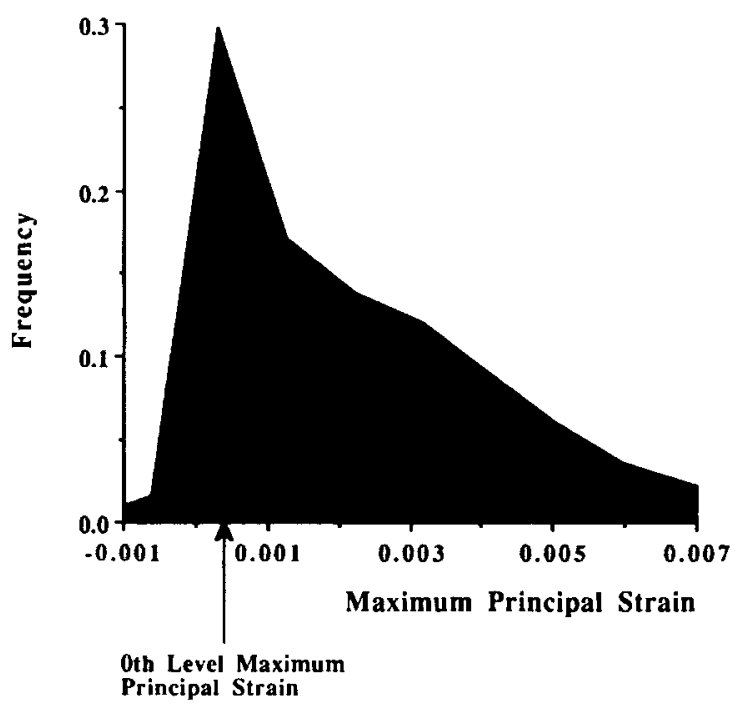

Figure 8. Histogram of frequency at which a given level of strain occurs within a $4 \times 4 \times 4 \mathrm{~mm}$ of first-level trabecular bone microstructure. The zero-level maximum principal strain of $495 \mu \mathrm{E}$ led to strains ranging between -1000 and over $+7000 \mu \mathrm{E}$ in the trabecular tissue. exhibited a much more uniform maximum principal strain distribution (Fig. 9). In each case, the maximum principal level strains ranged between 782 and $2530 \mu \mathrm{E}$ in tension. Lacunar orientation along principal strain directions also led to more uniform distributions of minimum principal strains (Fig. 10). Strains immediately around the lacunae rim were approximately double the average strain within the bone matrix. Note that since the idealized lacunae were modeled using smooth finite elements, the strain concentrations at the lacunae rim do not suffer from the digital image based mesh numerical oscillations.

The effect of osteocyte lacunar ellipsoidal geometry on both effective stiffness and local strains was much less significant than osteocyte lacunar orientation. Increasing the ellipticity from $1.5 / 1$ to $2 / 1$ slightly increased the ratio between material stiffness matrix elements $C_{22} / C_{11}$ from 1.01 to 1.04 . Increasing the ellipticity increased the strain concentration effect slightly. The largest maximum principal strains increased from 2530 to $2690 \mu \mathrm{E}$ in tension when the axes ratio of the ellipsoid increase from 1.5/1 to $2 / 1$. Orienting the lacunae along the principal strain directions produced a more uniform strain distribution around the lacuna similar to the $1.5 / 1$ ellipticity ratio.

\section{DISCUSSION}

This study has demonstrated the large strain variations going from the whole bone level down to the osteocyte lacunar level. Whole bone maximum principal strains of $495 \mu \mathrm{E}$ engendered osteocyte lacunar maximum principal strains ranging between 782 and $2530 \mu \mathrm{E}$. Each level of microstructure amplified the strain range from the next most macroscopic level. The more inhomogeneous the structure, the broader the amplification. The osteocyte lacunar level analysis suggests that strains will be highest around lacunae, or other holes such as canaliculi, within the bone matrix. Since large strain gradients exist around lacunae, osteocytes within lacunae would likely be more sensitive to changes in mechanical environment than cells on the bone surface. This fact would lend some credence to the hypothesis that osteocytes are strain sensors within the bone matrix.

An intriguing finding was the fact that lacunae oriented along principal strain directions within the tissue produced a uniform strain distribution compared with other lacunar orientations. It has been hypothesized that different levels of bone structure are oriented along the next most macroscopic level principal strain or stress directions. Both van Meyer in 1867 and Wolff in 1869, (reported in ref. 47), hypothesized that trabeculae were aligned along the zero-level principal stress directions within the whole bone. Martin and Burr ${ }^{41}$ hypothesized that osteons in cortical bone were formed along compressive principal strain directions. Martin and Burr suggested that strain gradients produced around newly forming osteons regulate the activity of osteoclasts and osteoblasts remodeling bone. Principal strain directions within the surrounding bone matrix may serve as a guide to direct bone deposition if the osteoblasts forming bone 

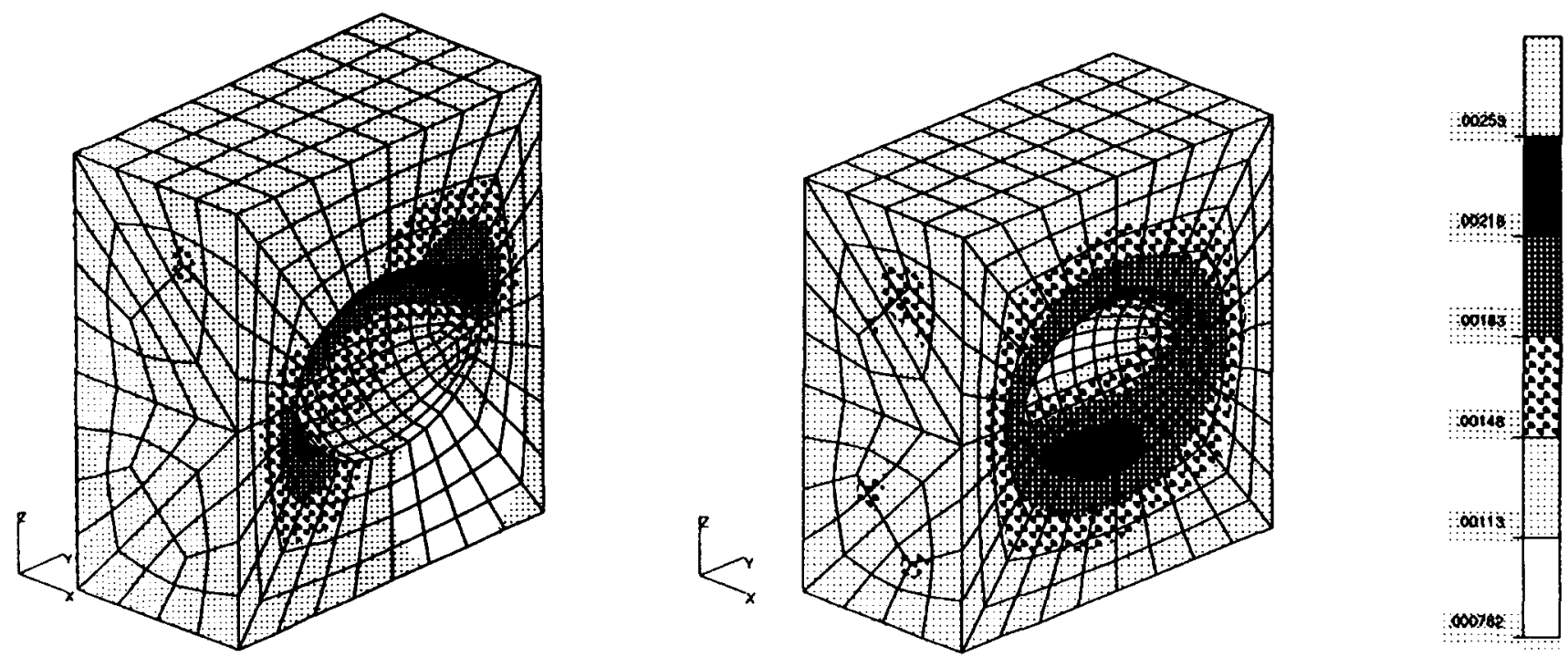

Figure 9. Maximum principal strain distributions around idealized osteocyte lacunae for arbitrary lacunar orientation (left) and for lacunae oriented along principal strain directions (right). Strains range between 782 and $2530 \mu \mathrm{E}$ for a first-level principal strain of $1325 \mu \mathrm{E}$.

seek to exist within the most uniform strain environment possible. It is interesting to note that Pedersen ${ }^{44}$ determined that orienting composite material microstructure along principal stress directions yields the most optimal structure. Principal strain directions may possibly provide a feedback mechanism directing bone cells to lay down bone matrix in an optimal manner.

The accuracy of homogenization RVE analyses will be limited by a number of factors including RVE boundary condition assumptions, lack of experimental stiffness data on bone microstructural components, inaccuracies in thresholding digital images of bone microstructure, and numerical artifacts resulting from nonsmooth finite element meshes made from digital images. Displacement boundary condition assumptions will theoretically lead to an underprediction of both effective stiffness and local strain. Hollister et al. ${ }^{29}$ found that digital image based homogenization analysis of trabecular bone tended to underestimate trabecular bone effective stiffness. Numerical artifacts have a limited effect at the boundary between dissimilar materials ${ }^{32}$ and may possibly be smoothed numerically a posteriori. Perhaps the biggest limiting factor encountered when analyzing bone microstructure is the uncertain relationship between measured stiffness and digital image voxel density. Whereas theoretical assumptions may lead to inaccuracies on the order of $50 \%$ to $70 \%,{ }^{34}$ uncertainties concerning experimental stiffness values may lead to inaccuracies well over $100 \%$ to $200 \%$. Although definitive accuracy of less than $10 \%$ is out of the reach at present, digital image based homogenization analysis has been shown to give consistent predictions which can be used to test hypotheses concerning bone adaptation. ${ }^{33}$
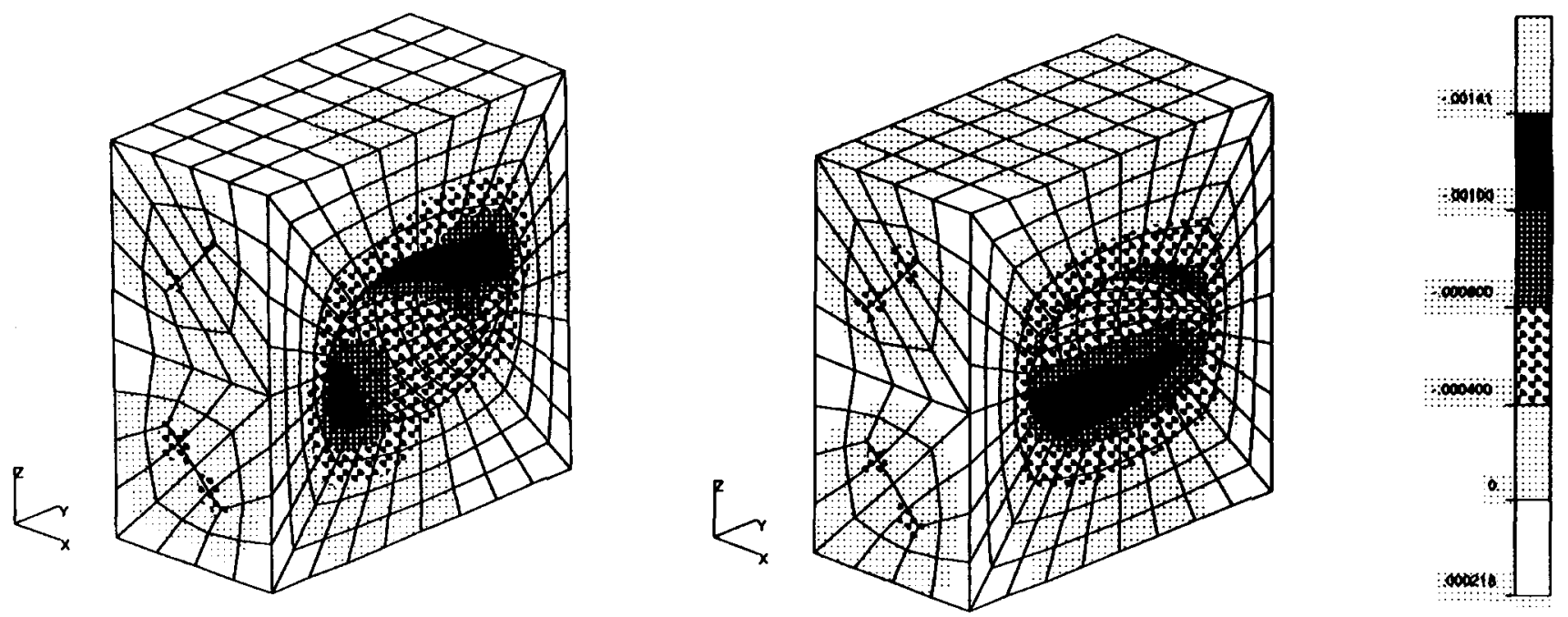

Figure 10. Minimum principal strain distributions around idealized osteocyte lacunae for arbitrary lacunar orientation (left) and for lacunae oriented along principal strain directions (right). Strains range between -1410 and $+218 \mu \mathrm{E}$. 
In conclusion, homogenization theory provides a systematic mathematical framework which can be fit to analyze the multiple level structure of bone. When combined with digital imaging of bone microstructure, homogenization theory can provide strain estimates for large numbers of specimens from experimental studies. Multiple level homogenization RVE based analysis can provide a bridge between whole bone and cell culture studies of adaptation by simulating load transfer from the whole bone level to the cell level. Cell level strain estimates from whole bone studies can then be used as input for cell culture experiments studying the biochemical pathways by which cells sense and respond to mechanical stimulus.

The authors gratefully acknowledge the substantial effort of Mr. Karl Jepsen of the Orthopaedic Research Laboratories at the University of Michigan for obtaining the scanning electron microscopy (SEM) of cortical and trabecular bone microstructure. We would also like to acknowledge the assistance of Dennis Kayner with the SEM. The first author is indebted to the Bone Remodeling Discussion Group at the Orthopaedic Research Laboratories for many thought-provoking discussions concerning bone adaptation studies. We also acknowledge the substantial input of Steven Goldstein and Robert Goulet. Finally, we thank the reviewers for their helpful comments. This work was supported by the National Institutes of Health (AR31793, AR34399; to S. J. H.), the Whitaker Foundation (to S.J.H.), and the National Science Foundation, Grant No. 9021683 (to N. K.)

\section{References}

1. Ascenzi, A., Bonucci, E. 1976. Mechanical similarities between alternate osteons and cross-ply laminates. J. Biomechan. 9: 65-71.

2. Ascenzi, A., Benvenuti, A. 1986. Orientation of collagen fibers at the boundary between two successive osteonic lamellae and its mechanical interpretation. J. Biomechan. 6: 455-463.

3. Babuska, I. 1976. Homogenization approach in engineering. pp. 137153. Proc. 2nd International Symposium on Computational Methods in Applied Science and Engineering. Springer-Verlag, Berlin.

4. Bakhvalov, N., Panasenko, G. 1989. Homogenisation: Averaging processes in periodic media. Kluwer Academic Publishers, Dordrecht, The Netherlands.

5. Beaupré, G.S., Hayes, W.C. 1985. Finite element analysis of a three dimensional open celled model for trabecular bone. J. Biomech. Eng. 107: $249-256$.

6. Brand, R. A. 1992. Autonomous informational stability in connective tissues. Med. Hypoth. 37: 107-114.

7. Burger, E. H., Veldhuijzen, J. P. 1993. Influence of mechanical factors on bone formation, resorption, and growth in vitro. pp. 37-56. In: B. K. Hall (ed.), Bone, volume 7: Bone growth-B. CRC Press, Boca Raton, FL.

8. Cané, V., Marotti, G., Volpi, G., Zaffe, D., Palazzini, S., Remaggi, F., Muglia, M. A. 1982. Size and density of osteocyte lacunae in different regions of long bones. Calcif. Tissue Int. 34: 558-563.

9. Carey, G. F., Jiang, B. N. 1986. Element-by-element linear and nonlinear solution schemes. Comm. App. Num. Meth. 2: 145-153.

10. Carter, D. R., Harris, W.H., Vasu, R., Caler, W.E. 1981. The mechanical and biological response of cortical bone to in vivo strain histories. pp. 81-92. In: S. C. Cowin (ed.), Mechanical properties of bone. ASME, New York. vol. 45.

11. Chamay, A., Tschantz, P. 1972. Mechanical influences in bone remodeling: Experimental research on Wolff's law. J. Biomech. 5: $173-180$.

12. Choi, K., Kuhn, J. L., Ciarelli, M. J., Goldstein, S. A. 1990. The elastic modulus of trabecular, subchondral, and cortical bone tissue and the size dependency of cortical bone modulus. J. Biomech. 23: $1103-1113$.

13. Choi, K., Goldstein, S. A. 1991. The fatigue properties of bone tissues on a microstructural level. Trans. 37th Annual Orthopaedic Research Society meeting, p. 485. Orthopaedic Research Society, Palatine, IL.

14. Crolet, J. M., Aoubiza, B., Meunier, A. 1993. Compact bone: Numerical simulation of mechanical characteristics. J. Biomech. 26: $677-689$.

15. Currey, J.D. 1964. Three analogies to explain the mechanical properties of bone. Biorheology 2: 1-10.

16. El Haj, A. J., Minter, S. L., Rawlinson, S.C., Suswillo, R., Lanyon, L. E. 1990. Cellular responses to mechanical loading in vitro. J. Bone Min. Res. 5: 923-932.

17. Feldkamp, L.A., Goldstein, S. A., Parfitt, A.M., Jesion, G., Kleerekoper, M. 1989. The direct examination of three dimensional bone architecture in vitro by computed tomography. J. Bone Min. Res. 4: 3-11.

18. Ferencz, R. M. 1990. Element-by-element preconditioning techniques for large-scale, vectorized finite element analysis in nonlinear solid and structural mechanics. Ph.D. Dissertation, Stanford University.

19. Frost, H. M. 1986. Intermediary organization of the skeleton, vol. 1. CRC Press, Boca Raton, FL.

20. Fyhrie, D.P., Hamid, M.S., Kuo, R.F., Lang, S. M. 1992. Direct three-dimensional finite element analysis of human vertebral cancellous bone. Trans. 38th Orthopaedic Research Society meeting, p. 551. Orthopaedic Research Society, Palatine, IL.

21. Gibson, L. J. 1985. The mechanical behavior of cancellous bone. J. Biomech. 18: 317-328.

22. Giraud-Guille, M. M. 1988. Twisted plywood architecture of collagen fibrils in human compact bone osteons. Calcif. Tissue Int. 42: $167-180$.

23. Harell, A., Dekel, S., Binderman, I. 1977. Biochemical effect of mechanical stress on cultured bone cells. Calcif. Tissue Res. 22S: 202-209.

24. Hasegawa, S., Sato, S., Saito, S., Suzuki, Y., Brunette, D. M. 1985. Mechanical stretching increases the number of cultured bone cells synthesizing DNA and alters their pattern of protein synthesis. Calcif. Tissue Res. 37: 431-436.

25. Hashin, Z. 1983. Analysis of composite materials - a survey. J. Appl. Mech. 50: 481-505.

26. Hashin, Z., Rosen, B.W. 1964. The elastic moduli of fiber reinforced materials. J. Appl. Mech. 31: 223-232.

27. Hill, R. 1963. Elastic properties of reinforced solids: Some theoretical principles. J. Mech. Phys. Solids. 11: 357-372.

28. Hogan, H. A. 1992. Micromechanics modeling of haversian cortical bone properties. J. Biomech. 25: 549-556.

29. Hollister, S. J., Brennan, J. M., Kikuchi, N. 1992. Homogenization sampling analysis of trabecular bone microstructural mechanics. pp. 308-317 In: J. Middleton, G. N. Pande, and K. R. Williams (eds.), Recent advances in computer methods in biomechanics and biomedical engineering. Books \& Journals Int., Swansea, United Kingdom.

30. Hollister, S. J., Fyhrie, D.P., Jepsen, K.J., Goldstein, S. A. 1991. Application of homogenization theory to the study of trabecular bone mechanics. J. Biomechan. 24: 825-839.

31. Hollister, S. J., Kikuchi, N. 1992. A comparison of homogenization and standard mechanics analyses for periodic porous composites. Comp. Mech. 10: 73-95.

32. Hollister, S. J., Riemer, B. A. 1993. Digital-image-based finite element analysis for bone microstructure using conjugate gradient and Gaussian filter techniques. Mathematical Methods in Medical Imaging II. Proceedings SPIE-The International Society for Optical Engineering, pp. 95-106. Society of Photo-Optical Instrumentation Engineers, Bellingham, WA.

33. Hollister, S.J., Goldstein, S.A. 1993. Trabecular tissue strain estimates for bone adapted to controlled implant loads. pp. 254-257 In: Proceedings 1993 Bioengineering Conference, ASME BED, vol. 24. ASME, New York. 
34. Hollister, S. J., Kikuchi, N. 1993. Comparison of trabecular tissue strains computed by homogenization theory to direct finite element calculations. pp. 258-261 In: Proceedings 1993 Bioengineering Conference, vol. 24. ASME, New York.

35. Jee, W.S.S. 1983. The skeletal tissue. pp. 206-254 In: L. Weiss (ed.), Histology: Cell and tissue biology, 5th edition.

36. Katz, J.L. 1981. Composite material models for cortical bone. pp. 171-184 In: S. C. Cowin (ed.), Mechanical properties of bone, vol. 45. ASME, New York.

37. Keller, J.B. 1977. Effective behavior of heterogenous media. pp. 631-644 In: Proc. Symposium on Statistical Mechanics and Statistical Methods. Plenum, New York.

38. Kikuchi, N. 1986. Finite element methods in mechanics. Cambridge University Press, Cambridge.

39. Larsen, E. W. 1975. Neutron transport and diffusion in inhomogeneous media. Int. J. Math. Phys. 16: 1421-1427.

40. Marotti, G., Muglia, M.A. 1988. A scanning electron microscope study of human bony lamellae: Proposal for a new model of collagen lamellar organization. Arch. Ital. Anat. Embriol. 93: $163-175$.

41. Martin, R. B., Burr, D. B. 1989. Structure, function, and adaptation of compact bone. Raven Press, New York

42. Murray, D.W., Rushton, N. 1990. The effect of strain on bone cell prostaglandin E2 release: A new experimental method. Calcif. Tissue Res. 47: 35-39.

43. Odgaard, A., Andersen, K., Melsen, F., Gundersen, H. J. G. 1990. A direct method for fast three-dimensional serial reconstruction. J. Microsc. 159: 335-342.

44. Pedersen, P. 1989. On optimal orientation of orthotropic materials. Structural Optimization 1: 101-106.

45. Pugh, J.W., Rose, R.M., Radin, E.L. 1973. A structural model for the mechanical behavior of trabecular bone. J. Biomechan. 6: $657-670$
46. Rawlinson, S. C. F., El-Haj, A. J., Minter, S. L., Tavares, I. A., Bennett, A., Lanyon, L.E. 1991. Loading-related increases in prostaglandin production in cores of adult canine cancellous bone in vitro: Role for prostacyclin in adaptive bone remodeling? J. Bone Min. Res. 6: $1345-1351$.

47. Roesler, H. 1981. Some historical remarks on the theory of cancellous bone structure (Wolff's law). pp. 13-26 In: S. C. Cowin (ed.), Mechanical properties of bone. vol. 45. ASME, New York.

48. Rubin, C. T., Lanyon, L.E. 1982. Limb mechanics as a function of speed and gait: A study of functional strains in the radius and tibia of horse and dog. J. Exp. Biol. 101: 187-211.

49. Sanchez-Palencia, E. 1974. Comportements local et macroscopique d'un type de milieux physiques heterogenes. Int. J. Eng. Sci. 12: 331-351.

50. Sanchez-Palencia, E. 1980. Non-homogeneous media and vibration theory. Springer-Verlag, Berlin.

51. Schaffler, M. B., Burr, D.B., Fredrickson, R. G. 1987. Morphology of the osteonal cement line in human bone. Anat. Rec. 217: $223-228$.

52. van Rietbergen, B., Weinans, H., Huiskes, R., Odgaard, A. 1993. Three dimensional analysis of a realistic trabecular bone structure using a large-scale fe-model. Proceedings 1993 Bioengineering Conference. vol. 24. ASME, New York, pp. 250-253.

53. Weiner, S., Traub, W. 1992. Bone structure: From angstroms to microns. The FASEB J. 6: 879-885.

54. Williams, J. L., Lewis, J. L. 1982. Properties and an anisotropic model of cancellous bone from the proximal tibia epiphysis. J. Biomech. Eng. 104: $50-56$.

55. Wolff, J. 1892. Das Gesetz der Transformation der Knochen. Hirschwald, Berlin.

56. Yeh, E. H. K., Rodan, G. A. 1984. Tensile forces enhance PGE synthesis in osteoblasts grown on collagen ribbon. Calcif. Tissue Res. 36S: $67-71$ 